KYUSHU-HET 47

\title{
Matter profile effect in neutrino factory
}

\author{
T. Ota \\ Department of Physics, Kyushu University, Fukuoka 812-8581, Japan \\ J. Sato \\ Research Center for Higher Education, Kyushu University, \\ Ropponmatsu, Chuo-ku, Fukuoka, 810-8560, Japan
}

\begin{abstract}
We point out that the matter profile effect — the effect of matter density fluctuation on the baseline - is very important to estimate the parameters in a neutrino factory with a very long baseline. To make it clear, we propose the method of the Fourier series expansion of the matter profile. By using this method, we can take account of both the matter profile effect and its ambiguity. For very long baseline experiment, such as $L=7332 \mathrm{~km}$, in the analysis of the oscillation phenomena we need to introduce a new parameter $a_{1}$ - the Fourier coefficient of the matter profile - as a theoretical parameter to deal with the matter profile effects.
\end{abstract}




\section{Introduction}

The evidence of the neutrino oscillation was presented by the Super-Kamiokande collaboration[回]. Many experiments have been planed and some of them have already been carried out to determine the parameters of the neutrino oscillation[2]. Recently, the neutrino factory [3] - a very long (over $1000 \mathrm{~km}$ ) baseline experiment with high energy neutrinos from a muon storage ring - has been proposed as the most effective experiment to determine the unknown parameters, $U_{e 3}$, the sign of $\Delta m^{2}$ which is responsible for the atmospheric neutrino anomaly and the $\mathrm{CP}$ violating phase 4, 5, 6, 0, 8, 9, 10, 11, 12, 13.

To analyze the oscillation phenomena with such a long baseline, we have to treat the matter effect 14 very carefully. Two ways have been adopted to deal with the matter effects: (i) To add the averaged matter density $\bar{\rho}$ as a parameter in addition to the other theoretical parameters like the mixing angles, and estimate it in the same way as the other parameters [5, 9, 11]. (ii) To use the PREM-Preliminary Reference Earth Model 15 for the matter density profile, and to assume that we know the ingredients of the earth completely [4, 6]. We have, however, questions to these treatments. For the method-(i), "Can we describe the matter effect precisely enough by only averaged matter density ?" The answer was "No, we can't. Sometimes the deviation from the constant density is important." [4]. For the method-(ii), "Is the PREM a trustworthy model for the neutrino oscillation experiments ?" The PREM is originally based on the study of earthquake waves, hence it predicts the density profile in the depth However, it doesn't predict the ingredients of the matter. Therefore, there is an ambiguity in electron number fraction. Then, we should worry whether there are ambiguities in electron number density. If we estimated the parameters without considering its ambiguity, we would have significant errors in the estimates for the parameters. Therefore, we must introduce the way to take into account the matter profile effect as the parameter determined by the experiments. We propose the method of the Fourier series expansion of the matter profile 16. Using the Fourier expansion we can parameterize the matter profile. We can express the matter profile effect with a finite number of the parameters by examining how many terms of the Fourier expansion contribute to the oscillation physics within the resolution of the experiments. We can incorporate the matter profile's ambiguity in the ambiguities of the Fourier coefficients.

We will review the method of the Fourier series and investigate qualitatively what circumstances make the matter profile effect relevant in section 2 . In section 3 , we will calculate quantitatively the oscillation probabilities and the event rates with various sets of the parameters in baseline lengths.

\section{The method of the Fourier series}

In this section we introduce the Fourier expansion method. First, we describe its formalism and see its feature. Then, we solve the evolution equation for neutrinos in matter perturbatively, and study the condition where the matter profile effect is significant. 


\section{$2.1 \quad$ Fourier expansion method}

Assuming three generations of neutrinos, we parameterize the lepton mixing matrix

$$
\begin{aligned}
U_{\alpha i} & \equiv e^{i \psi \lambda_{7}} \Gamma e^{i \phi \lambda_{5}} e^{i \omega \lambda_{2}} U_{\text {Majorana }} \\
& =\left(\begin{array}{ccc}
1 & & \\
& c_{\psi} & s_{\psi} \\
& -s_{\psi} & c_{\psi}
\end{array}\right)\left(\begin{array}{ccc}
1 & & \\
& 1 & \\
& & e^{i \delta}
\end{array}\right)\left(\begin{array}{ccc}
c_{\phi} & & s_{\phi} \\
& 1 & \\
-s_{\phi} & & c_{\phi}
\end{array}\right)\left(\begin{array}{ccc}
c_{\omega} & s_{\omega} & \\
-s_{\omega} & c_{\omega} & \\
& & 1
\end{array}\right) U_{\text {Majorana }} \\
& =\left(\begin{array}{ccc}
c_{\phi} c_{\omega} & c_{\phi} s_{\omega} & \\
-c_{\psi} s_{\omega}-s_{\psi} s_{\phi} c_{\omega} e^{i \delta} & c_{\psi} c_{\omega}-s_{\psi} s_{\phi} s_{\omega} e^{i \delta} & s_{\psi} c_{\phi} e^{i \delta} \\
s_{\psi} s_{\omega}-c_{\psi} s_{\phi} c_{\omega} e^{i \delta} & -s_{\psi} c_{\omega}-c_{\psi} s_{\phi} s_{\omega} e^{i \delta} & c_{\psi} c_{\phi} e^{i \delta}
\end{array}\right) U_{\text {Majorana }} .
\end{aligned}
$$

which relates the flavor eigenstates $\left|\nu_{\alpha}\right\rangle(\alpha=e, \mu, \tau)$ with the mass eigenstates in vacuum $\left|\nu_{i}\right\rangle(i=1,2,3)$ as

$$
\left|\nu_{\alpha}\right\rangle=U_{\alpha i}\left|\nu_{i}\right\rangle .
$$

$U_{\text {Majorana }}$ is the part of the Majorana phases. It doesn't contribute to the neutrino oscillation phenomena and hence is omitted hereafter.

The evolution in matter of the flavor eigenstates of neutrinos] with its energy $E$ is given by

$$
\begin{aligned}
i \frac{d}{d x}\left|\nu_{\beta}(x)\right\rangle & =H(x)_{\beta \alpha}\left|\nu_{\alpha}(x)\right\rangle \\
H(x)_{\beta \alpha} & \equiv \frac{1}{2 E}\left\{U_{\beta i}\left(\begin{array}{lll}
0 & & \\
& \Delta m_{21}^{2} & \\
& & \Delta m_{31}^{2}
\end{array}\right) U_{i \alpha}^{\dagger}+\left(\begin{array}{ccc}
a(x) & & \\
& 0 & \\
& & 0
\end{array}\right)\right\},
\end{aligned}
$$

where

$$
\begin{aligned}
& \Delta m_{i j}^{2} \equiv m_{i}^{2}-m_{j}^{2}, \quad m_{i} \text { : mass eigenvalue } \\
& a(x) \equiv 2 \sqrt{2} G_{F} n_{e}(x) E=7.56 \times 10^{-5}\left(\frac{\rho(x)}{\mathrm{g} / \mathrm{cm}^{3}}\right)\left(\frac{Y_{e}}{0.5}\right)\left(\frac{E}{\mathrm{GeV}}\right)\left[\mathrm{eV}^{2}\right] \\
& G_{F} \quad: \text { Fermi constant } \\
& n_{e}(x) \text { : electron number density } \\
& \rho(x) \quad \text { : matter density } \\
& Y_{e} \quad \text { : electron fraction }
\end{aligned}
$$

To parameterize the matter effect, $a(x)$, we expand $a(x)$ into Fourier series as

$$
a(x)=\sum_{n=-\infty}^{\infty} a_{n} e^{-i p_{n} x}, \quad p_{n} \equiv \frac{2 \pi}{L} n .
$$

\footnotetext{
${ }^{1}$ For antineutrino $a(x)$ and $\delta$ should be replaced by $-a(x)$ and $-\delta$.
} 
Note that $a_{-n}=a_{n}^{*}$ due to the reality of $a(x)$. Also within the PREM,

$$
a_{-n}=a_{n}
$$

since $a(x)=a(L-x)$.

If this expansion can be approximated with a finite number $(\equiv N)$ of terms,

$$
a(x)=\sum_{n=-N}^{n=N} a_{n} e^{-i p_{n} x},
$$

then it means that the matter profile effect can be parametrized. Thus, by introducing new parameters $a_{n}(n=-N, \cdots, N)$ (or $n=0, \cdots, N$, if eq.(6) holds), we can investigate the oscillation physics without help of earth models.

\subsection{Perturbative analysis of matter profile effect}

We solve the evolution equation (3) to see the qualitative feature of the matter profile effect. First, we divide the Hamiltonian $H(x)$ into three pieces for the later calculation:

$$
\begin{aligned}
& H_{00} \equiv \frac{1}{2 E} e^{i \psi \lambda_{7}} \Gamma\left(\begin{array}{ccc}
\Delta m_{31}^{2} s_{\phi}^{2}+\bar{a}+\Delta m_{21}^{2} c_{\phi}^{2} s_{\omega}^{2} & 0 & \Delta m_{31}^{2} c_{\phi} s_{\phi}-\Delta m_{21}^{2} c_{\phi} s_{\phi} s_{\omega}^{2} \\
0 & \Delta m_{21} c_{\omega}^{2} & 0 \\
\Delta m_{31}^{2} c_{\phi} s_{\phi}-\Delta m_{21}^{2} c_{\phi} s_{\phi} s_{\omega}^{2} & 0 & \Delta m_{31}^{2} c_{\phi}^{2}+\Delta m_{21} s_{\phi}^{2} s_{\omega}^{2}
\end{array}\right) \Gamma^{\dagger} e^{-i \psi \lambda_{7}}, \\
& H_{01} \equiv \frac{\Delta m_{21}^{2}}{2 E} c_{\omega} s_{\omega} e^{i \psi \lambda_{7}} \Gamma\left(\begin{array}{ccc}
0 & c_{\phi} & 0 \\
c_{\phi} & 0 & -s_{\phi} \\
0 & -s_{\phi} & 0
\end{array}\right) \Gamma^{\dagger} e^{-i \psi \lambda_{7}} \\
& H_{1}(x) \equiv \frac{1}{2 E}\left(\begin{array}{ccc}
\delta a(x) & & \\
& 0 & \\
& & 0
\end{array}\right) \text {, }
\end{aligned}
$$

where $\bar{a} \equiv a_{0}$ and $\delta a(x) \equiv a(x)-\bar{a}$. The oscillation phenomena arise from $H_{00}$, hence its effect must be fully taken into account. On the other hand $H_{01}$ and $H_{1}(x)$ can be treated as the perturbation in almost all cases in neutrino factories, since

$$
\left|\frac{\Delta m_{21}^{2} L}{2 E}\right| \ll 1 \quad \text { and } \quad\left|\frac{\delta a(x) L}{2 E}\right| \ll 1
$$

can be almost always expected. Note that we have to include $\Delta m_{21}^{2}$ terms in $\left(H_{00}\right)_{i j}(i, j=$ 1,3) to deal with the resonance effect.?

Next, we rewrite $H_{00}$ as

$$
H_{00}=\frac{1}{2 E} \tilde{U}_{0}\left(\begin{array}{lll}
\lambda_{-} & & \\
& \Delta m_{21}^{2} c_{\omega}^{2} & \\
& & \lambda_{+}
\end{array}\right) \tilde{U}_{0}^{\dagger}
$$

\footnotetext{
${ }^{2}$ On the other hand, we include $\Delta m_{21}^{2}$ term in the (22) element just for convenience. This inclusion is not essential.
} 
where $\tilde{U}_{0}$ and $\lambda_{ \pm}$are the mixing matrix and mass square eigenvalues in matter up to the zeroth order of the "perturbation"s $H_{01}$ and $H_{1}(x)$ and defined by

$$
\begin{aligned}
\tilde{U}_{0} & \equiv e^{i \psi \lambda_{7}} \Gamma e^{i \tilde{\phi} \lambda_{5}}=\left(\begin{array}{ccc}
c_{\tilde{\phi}} & 0 & s_{\tilde{\phi}} \\
-s_{\psi} s_{\tilde{\phi}} e^{i \delta} & c_{\psi} & s_{\psi} c_{\tilde{\phi}} e^{i \delta} \\
-c_{\psi} s_{\tilde{\phi}} e^{i \delta} & s_{\psi} & c_{\psi} c_{\tilde{\phi}} e^{i \delta}
\end{array}\right) \\
\tan 2 \tilde{\phi} & =\frac{s_{2 \phi}\left(\Delta m_{31}^{2}-\Delta m_{21}^{2} s_{\omega}^{2}\right)}{c_{2 \phi}\left(\Delta m_{31}^{2}-\Delta m_{21}^{2} s_{\omega}^{2}\right)-\bar{a}}, \\
\lambda_{ \pm} & =\frac{1}{2}\{\alpha \pm \beta\} \\
\alpha & \equiv \Delta m_{31}^{2}+\Delta m_{21}^{2} s_{\omega}^{2}+\bar{a}, \\
\beta & \equiv \sqrt{\left\{\left(\Delta m_{31}^{2}-\Delta m_{21}^{2} s_{\omega}^{2}\right) c_{2 \phi}-\bar{a}\right\}^{2}+\left(\Delta m_{31}^{2}-\Delta m_{21}^{2} s_{\omega}^{2}\right)^{2} s_{2 \phi}^{2}} .
\end{aligned}
$$

Then we solve the evolution equation (3) perturbatively as

$$
\begin{aligned}
\left|\nu_{\beta}(L)\right\rangle & =\mathrm{T}\left[\exp \left(-i \int_{0}^{L} H(x) d x\right)\right]_{\beta \alpha}\left|\nu_{\alpha}(0)\right\rangle \\
& =\left(e^{-i H_{00} L}+e^{-i H_{00} L}(-i) \int_{0}^{L} H_{01 I} d x+e^{-i H_{00} L}(-i) \int_{0}^{L} H_{1}(x)_{I} d x+\cdots\right)_{\beta \alpha}\left|\nu_{\alpha}(0)\right\rangle \\
& \equiv\left(S_{00}+S_{01}+S_{1}+\cdots\right)_{\beta \alpha}\left|\nu_{\alpha}(0)\right\rangle
\end{aligned}
$$

where $S_{00}, S_{01}$ and $S_{1}$ are defined by the first, second and third terms in the second last line respectively. $S_{01}$ and $S_{1}$ are the perturbative contributions at the first order from $H_{01}$ and $H_{1}$ respectively. $S_{00}, S_{01}$ and $S_{1}$ are calculated to be

$$
\begin{aligned}
& S_{00}(L)_{\beta \alpha} \equiv e^{-i H_{00} L} \\
& =\tilde{U}_{0}\left(\begin{array}{ccc}
e^{-i \frac{\lambda_{-}}{2 E} L} & & \\
& e^{-i \frac{\Delta m_{21}^{2} c_{\omega}^{2}}{2 E} L} & \\
& & e^{-i \frac{\lambda_{+}}{2 E} L}
\end{array}\right) \tilde{U}_{0}^{\dagger} \\
& =\left(\begin{array}{cc}
s_{\tilde{\phi}}^{2} e^{-i \frac{\lambda_{+}}{2 E} L}+c_{\tilde{\phi}}^{2} e^{-i \frac{\lambda_{-}}{2 E} L} & \frac{1}{2} e^{-i \delta} s_{\psi} s_{2 \tilde{\phi}}\left(e^{-i \frac{\lambda_{+}}{2 E} L}-e^{-i \frac{\lambda_{-}}{2 E} L}\right) \\
\frac{1}{2} e^{i \delta} s_{\psi} s_{2 \tilde{\phi}}\left(e^{-i \frac{\lambda_{+}}{2 E} L}-e^{-i \frac{\lambda_{-}}{2 E} L}\right) & s_{\psi}^{2}\left(c_{\tilde{\phi}}^{2} e^{-i \frac{\lambda_{+}}{2 E} L}+s_{\tilde{\phi}}^{2} e^{-i \frac{\lambda_{-}}{2 E} L}\right)+c_{\psi}^{2} e^{-i \frac{\Delta m_{21} c_{\omega}^{2}}{2 E} L} \\
\frac{1}{2} e^{i \delta} c_{\psi} s_{2 \tilde{\phi}}\left(e^{-i \frac{\lambda_{+}}{2 E} L}-e^{-i \frac{\lambda_{-}}{2 E} L}\right) & \frac{1}{2} s_{2 \psi}\left(c_{\tilde{\phi}}^{2} e^{-i \frac{\lambda_{+}}{2 E} L}+s_{\tilde{\phi}}^{2} e^{-i \frac{\lambda_{-}}{2 E} L}-e^{-i \frac{\Delta m_{21} c_{\omega}^{2}}{2 E} L}\right)
\end{array}\right. \\
& \left.\begin{array}{c}
\frac{1}{2} e^{-i \delta} c_{\psi} s_{2 \tilde{\phi}}\left(e^{-i \frac{\lambda_{+}}{2 E} L}-e^{-i \frac{\lambda_{-}}{2 E} L}\right) \\
\left(c_{\tilde{\phi}}^{2} e^{-i \frac{\lambda_{+}}{2 E} L}+s_{\tilde{\phi}}^{2} e^{-i \frac{\lambda_{-}}{2 E} L}-e^{-i \frac{\Delta m_{21} c_{\omega}^{2}}{2 E} L}\right) \\
\left.{ }_{\tilde{\phi}}^{2} e^{-i \frac{\lambda_{+}}{2 E} L}+s_{\tilde{\phi}}^{2} e^{-i \frac{\lambda_{-}}{2 E} L}\right)+s_{\psi}^{2} e^{-i \frac{\Delta m_{21} c_{\omega}^{2}}{2 E} L}
\end{array}\right), \\
& S_{01}(L)_{\beta \alpha} \equiv e^{-i H_{00} L}(-i) \int_{0}^{L} d x H_{01 I}
\end{aligned}
$$




$$
\begin{aligned}
& =\tilde{U}_{0}\left(\begin{array}{ccc}
0 & \mathcal{A} & 0 \\
\mathcal{A} & 0 & \mathcal{B} \\
0 & \mathcal{B} & 0
\end{array}\right) \tilde{U}_{0}^{\dagger} \\
& =\left(\begin{array}{ccc}
0 & c_{\psi}\left(c_{\tilde{\phi}} \mathcal{A}+s_{\tilde{\phi}} \mathcal{B}\right) & -s_{\psi}\left(c_{\tilde{\phi}} \mathcal{A}+s_{\tilde{\phi}} \mathcal{B}\right) \\
c_{\psi}\left(c_{\tilde{\phi}} \mathcal{A}+s_{\tilde{\phi}} \mathcal{B}\right) & -s_{2 \psi}\left(s_{\tilde{\phi}}^{\mathcal{A}}-c_{\tilde{\phi}} \mathcal{B}\right) c_{\delta} & \left(e^{i \delta} s_{\psi}^{2}-e^{-i \delta} c_{\psi}^{2}\right)\left(s_{\tilde{\phi}} \mathcal{A}-c_{\tilde{\phi}} \mathcal{B}\right) \\
-s_{\psi}\left(c_{\tilde{\phi}} \mathcal{A}+s_{\tilde{\phi}} \mathcal{B}\right) & -\left(e^{i \delta} c_{\psi}^{2}-e^{-i \delta} s_{\psi}^{2}\right)\left(s_{\tilde{\phi}} \mathcal{A}-c_{\tilde{\phi}} \mathcal{B}\right) & s_{2 \psi}\left(s_{\tilde{\phi}} \mathcal{A}-c_{\tilde{\phi}} \mathcal{B}\right) c_{\delta}
\end{array}\right)
\end{aligned}
$$

$$
\begin{aligned}
\mathcal{A} & \equiv \frac{\Delta m_{21}^{2}}{2\left(\Delta m_{21}^{2} c_{\omega}^{2}-\lambda_{-}\right)} c_{\phi-\tilde{\phi}} s_{2 \omega}\left(e^{-i \frac{\Delta m_{21}^{2} c_{\omega}^{2}}{2 E} L}-e^{-i \frac{\lambda_{-}}{2 E} L}\right) \\
\mathcal{B} & \equiv \frac{\Delta m_{21}^{2}}{2\left(\lambda_{+}-\Delta m_{21}^{2} c_{\omega}^{2}\right)} s_{\phi-\tilde{\phi}} s_{2 \omega}\left(e^{-i \frac{\Delta m_{21}^{2} c_{\omega}^{2}}{2 E} L}-e^{-i \frac{\lambda_{+}}{2 E} L}\right)
\end{aligned}
$$

$$
\begin{aligned}
& S_{1}(L)_{\beta \alpha} \\
& \equiv e^{-i H_{00} L}(-i) \int_{0}^{L} d x H_{1}(x)_{I} \\
& =\sum_{n} \tilde{U}_{0}\left(\begin{array}{ccc}
0 & 0 & \mathcal{C}_{n} \\
0 & 0 & 0 \\
\mathcal{D}_{n} & 0 & 0
\end{array}\right) \tilde{U}_{0}^{\dagger} \\
& =\sum_{n}\left(\begin{array}{ccc}
\left(\mathcal{C}_{n}+\mathcal{D}_{n}\right) s_{\tilde{\phi}} c_{\tilde{\phi}} & e^{-i \delta_{1}} s_{\psi}\left(-\mathcal{D}_{n} s_{\tilde{\phi}}^{2}+\mathcal{C}_{n} c_{\tilde{\phi}}^{2}\right) & e^{-i \delta} c_{\psi}\left(-\mathcal{D}_{n} s_{\tilde{\phi}}^{2}+\mathcal{C}_{n} c_{\tilde{\phi}}^{2}\right) \\
e^{i \delta} s_{\psi}\left(\mathcal{D}_{n} c_{\tilde{\phi}}^{2}+\mathcal{C}_{n} s_{\tilde{\phi}}^{2}\right) & -\left(\mathcal{C}_{n}+\mathcal{D}_{n}\right) s_{\psi}^{2} s_{\tilde{\phi}} c_{\tilde{\phi}} & -\left(\mathcal{C}_{n}+\mathcal{D}_{n}\right) s_{\psi} c_{\psi} s_{\tilde{\phi}} c_{\tilde{\phi}} \\
e^{i \delta} c_{\psi}\left(\mathcal{D}_{n} c_{\tilde{\phi}}^{2}+\mathcal{C}_{n} s_{\tilde{\phi}}^{2}\right) & -\left(\mathcal{C}_{n}+\mathcal{D}_{n}\right) s_{\psi} c_{\psi} s_{\tilde{\phi}} c_{\tilde{\phi}} & -\left(\mathcal{C}_{n}+\mathcal{D}_{n}\right) c_{\psi}^{2} s_{\tilde{\phi}} c_{\tilde{\phi}}
\end{array}\right) \\
& =\sum_{n} a_{n} \frac{s_{2 \tilde{\phi}}\left(e^{-i \frac{\lambda_{+}}{2 E} L}-e^{-i \frac{\lambda_{-}}{2 E} L}\right)}{2\left\{\left(\lambda_{+}-\lambda_{-}\right)^{2}-\left(2 E p_{n}\right)^{2}\right\}} \\
& \times\left(\begin{array}{ccc}
\left(\lambda_{+}-\lambda_{-}\right) s_{2 \tilde{\phi}} & e^{-i \delta} s_{\psi}\left\{\left(\lambda_{+}-\lambda_{-}\right) c_{2 \tilde{\phi}}-2 E p_{n}\right\} & e^{-i \delta} c_{\psi}\left\{\left(\lambda_{+}-\lambda_{-}\right) c_{2 \tilde{\phi}}-2 E p_{n}\right\} \\
e^{i \delta} s_{\psi}\left\{\left(\lambda_{+}-\lambda_{-}\right) c_{2 \tilde{\phi}}+2 E p_{n}\right\} & -\left(\lambda_{+}-\lambda_{-}\right) s_{\psi}^{2} s_{2 \tilde{\phi}} & \frac{1}{2}\left(\lambda_{+}-\lambda_{-}\right) s_{2 \psi} s_{2 \tilde{\phi}} \\
e^{i \delta} c_{\psi}\left\{\left(\lambda_{+}-\lambda_{-}\right) c_{2 \tilde{\phi}}+2 E p_{n}\right\} & \frac{1}{2}\left(\lambda_{+}-\lambda_{-}\right) s_{2 \psi} s_{2 \tilde{\phi}} & -\left(\lambda_{+}-\lambda_{-}\right) c_{\psi}^{2} s_{2 \tilde{\phi}}
\end{array}\right), \\
& \mathcal{C}_{n} \equiv \frac{a_{n}}{2\left(\lambda_{+}-\lambda_{-}+2 E p_{n}\right)} s_{2 \tilde{\phi}}\left(e^{-i \frac{\lambda_{+}}{2 E} L}-e^{-i \frac{\lambda_{-}}{2 E} L}\right)=\mathcal{D}_{-n} \\
& \mathcal{D}_{n} \equiv \frac{a_{n}}{2\left(\lambda_{+}-\lambda_{-}-2 E p_{n}\right)} s_{2 \tilde{\phi}}\left(e^{-i \frac{\lambda_{+}}{2 E} L}-e^{-i \frac{\lambda_{-}}{2 E} L}\right)=\mathcal{C}_{-n} \text {. }
\end{aligned}
$$

With eq.(17), (18) and (21), the oscillation probability, for example from $\nu_{e}$ to $\nu_{\mu}$, up to the lowest order of the perturbations, $H_{01}$ and $H_{1}(x)$, is calculated as

$$
\begin{aligned}
P_{\nu_{e} \rightarrow \nu_{\mu}} & =\left|S(L)_{\mu e}\right|^{2} \\
& \simeq\left|\left(S_{00}\right)_{\mu e}\right|^{2}+2 \operatorname{Re}\left[\left(S_{00}\right)_{\mu e}\left(S_{01}\right)_{\mu e}^{*}\right]+2 \operatorname{Re}\left[\left(S_{00}\right)_{\mu e}\left(S_{1}\right)_{\mu e}^{*}\right]
\end{aligned}
$$




$$
\begin{aligned}
& =s_{\psi}^{2} s_{2 \tilde{\phi}}^{2} \sin ^{2} \frac{\lambda_{+}-\lambda_{-}}{4 E} L \\
& +\frac{1}{2} c_{\delta} s_{2 \psi} s_{2 \omega} s_{2 \tilde{\phi}} \\
& \times\left[\left(c_{\tilde{\phi}} c_{\phi-\tilde{\phi}} \frac{\Delta m_{21}^{2}}{\Delta m_{21}^{2} c_{\omega}^{2}-\lambda_{-}}+s_{\tilde{\phi}} s_{\phi-\tilde{\phi}} \frac{\Delta m_{21}^{2}}{\lambda_{+}-\Delta m_{21}^{2} c_{\omega}^{2}}\right) \sin ^{2} \frac{\Delta m_{21}^{2} c_{\omega}^{2}-\lambda_{-}}{4 E} L\right. \\
& -\left(c_{\tilde{\phi}} c_{\phi-\tilde{\phi}} \frac{\Delta m_{21}^{2}}{\Delta m_{21}^{2} c_{\omega}^{2}-\lambda_{-}}+s_{\tilde{\phi}} s_{\phi-\tilde{\phi}} \frac{\Delta m_{21}^{2}}{\lambda_{+}-\Delta m_{21}^{2} c_{\omega}^{2}}\right) \sin ^{2} \frac{\lambda_{+}-\Delta m_{21}^{2} c_{\omega}^{2}}{4 E} L \\
& \left.+\left(c_{\tilde{\phi}} c_{\phi-\tilde{\phi}} \frac{\Delta m_{21}^{2}}{\Delta m_{21}^{2} c_{\omega}^{2}-\lambda_{-}}-s_{\tilde{\phi}} s_{\phi-\tilde{\phi}} \frac{\Delta m_{21}^{2}}{\lambda_{+}-\Delta m_{21}^{2} c_{\omega}^{2}}\right) \sin ^{2} \frac{\lambda_{+}-\lambda_{-}}{4 E} L\right] \\
& +\frac{1}{4} s_{\delta} s_{2 \psi} s_{2 \omega} s_{2 \tilde{\phi}}\left(c_{\tilde{\phi}} c_{\phi-\tilde{\phi}} \frac{\Delta m_{21}^{2}}{\Delta m_{21}^{2} c_{\omega}^{2}-\lambda_{-}}+s_{\tilde{\phi}} s_{\phi-\tilde{\phi}} \frac{\Delta m_{21}^{2}}{\lambda_{+}-\Delta m_{21}^{2} c_{\omega}^{2}}\right) \\
& \times\left(\sin \frac{\lambda_{+}-\Delta m_{21}^{2} c_{\omega}^{2}}{2 E} L+\sin \frac{\Delta m_{21}^{2} c_{\omega}^{2}-\lambda_{-}}{2 E} L-\sin \frac{\lambda_{+}-\lambda_{-}}{2 E} L\right) \\
& +2 s_{\psi}^{2} s_{2 \tilde{\phi}}^{2} \sum_{n} a_{n}^{*} \frac{\left(\lambda_{+}-\lambda_{-}\right) c_{2 \tilde{\phi}}+2 E p_{n}}{\left(\lambda_{+}-\lambda_{-}\right)^{2}-\left(2 E p_{n}\right)^{2}} \sin ^{2} \frac{\lambda_{+}-\lambda_{-}}{4 E} L .
\end{aligned}
$$

From this equation (24) we find the following; (i) The matter profile effect is relevant when some of the Fourier coefficients $a_{n}$ 's are as large as $\Delta m_{21}^{2}$ or the resonance condition 17,

$$
\lambda_{+}-\lambda_{-}=2 E p_{n},
$$

is satisfied. (ii) The matter profile effect decreases in proportion to $1 / n$ when the resonance condition is not satisfied. Therefore the higher Fourier modes are expected to be irrelevant. In other words, we don't need the detail of the matter profile. Thus we expect that we can truncate the Fourier expansion of $a(x)$ at the finite number $N$. We can estimate the matter profile effect in neutrino factories.

\section{Numerical analysis}

In this section we examine how many terms in the Fourier expansion are necessary. To see this we compare the oscillation probability and the event rate calculated using the PREM with those calculated using the truncated Fourier series (7). We first calculate the transition probabilities with several matter profiles, the PREM matter profile and the Fourier series matter profiles that are truncated at various $N$ 's. Next using these probabilities we derive the event rates in the appearance channel, $\nu_{e} \rightarrow \nu_{\mu}$ as the "observed" numbers in an experiment. To derive them we use the following equation with a given number of muons in decay $N_{\mu}$, muon energy $E_{\mu}$, detector size $N_{k t}$ and detection efficiency $\epsilon$.

$$
N_{\nu_{e} \rightarrow \nu_{\mu}}=\int_{E_{t h}}^{E_{\mu}} \frac{d E}{E_{\mu}} \times \overbrace{\frac{N_{\mu}}{\pi L^{2}} \gamma^{2} 12\left(\frac{E}{E_{\mu}}\right)^{2}\left(1-\frac{E}{E_{\mu}}\right)}^{\text {flux }} \times \overbrace{P_{\nu_{e} \rightarrow \nu_{\mu}}(E)}^{\text {probability }} \times \overbrace{\sigma_{\nu}(E) N_{k t} N_{A} \epsilon}^{\text {detector }}
$$




$$
\begin{gathered}
\sigma_{\nu}=0.67 \times 10^{-42} \times\left(\frac{E}{\mathrm{GeV}}\right)\left[\mathrm{m}^{2}\right]: \text { CC cross section } \\
N_{A}: \text { Avogadro's number } \\
\gamma \equiv \frac{E_{\mu}}{m_{\mu}}, \quad m_{\mu}: \text { muon mass } \\
E_{t h}: \text { threshold energy of the detection. }
\end{gathered}
$$

Then we compare these results. Through these comparisons we find how many Fourier coefficients we have to introduce to the theoretical parameters.

In the following we assume that $N_{\mu} \times N_{\mathrm{kt}} \times \epsilon$ equals to $=5 \times 10^{22} \mathrm{kt}$.

\section{$3.1 \quad L=3000 \mathrm{~km}$}

Neutrino factories of this length are considered to be most efficient, so we consider this case first.

The matter profilef and its Fourier coefficients are shown in Fig.11. From these figures we find that the density fluctuation is very small. It should also be noted that the resonance condition (25) will not be satisfied for any $n$ in neutrino factories. These facts indicate that the the constant matter density approximation is valid. In other words, the Fourier modes will be irrelevant. To confirm this we plot the oscillation probabilities in Fig.2 with the various matter profiles; (i) constant density (dotted line), (ii) eq.(7) with $\mathrm{N}=1$ (dashed line), and (iii) PREM matter profile in Fig.1 (solid line). We find that indeed this figure confirms our expectation.

The event rates are shown in Fig.3. We plot the event rates calculated with the following $\delta$ 's and the matter profiles; (i) $\delta=\pi / 2$ and constant density (dotted line), (ii) $\delta=\pi / 2$ and the PREM matter profile (solid line), and (iii) $\delta=0$ and the PREM matter profile (dash-dotted line). The event rate with $\delta=\pi / 2$ is different from that with $\delta=0$. We can observe this difference, if we know all the other theoretical parameters, the mixing angles, $\Delta m^{2}$ 's and $\bar{a}$ accurately enough . Therefore we have to consider the contribution of $\delta$ to the oscillation probability. However, we cannot see the difference of the event rate in the case that $a_{1}=0$ and that $a_{1} \neq 0$ with baseline $L=3000 \mathrm{~km}$. Thus we do not have to take into account the fluctuation of the matter density.

\section{$3.2 \quad L=7332 \mathrm{~km}$}

There are many analyses of the oscillation physics with the baseline length $L=7332 \mathrm{~km}$ too. Therefore we consider this case next.

\footnotetext{
${ }^{3}$ We also assume that we can reconstruct neutrino energy.

4 To calculate the matter profile, we linearly interpolate the PREM's data given discretely for the depth. For this calculation we modify the density of the crust(= most outside layer) into $2.3 \mathrm{~g} / \mathrm{cm}^{3}$ from $1 \mathrm{~g} / \mathrm{cm}^{3}$.

${ }^{5}$ We cannot see this difference, however, since we will not determine the theoretical parameters accurately enough 18
} 


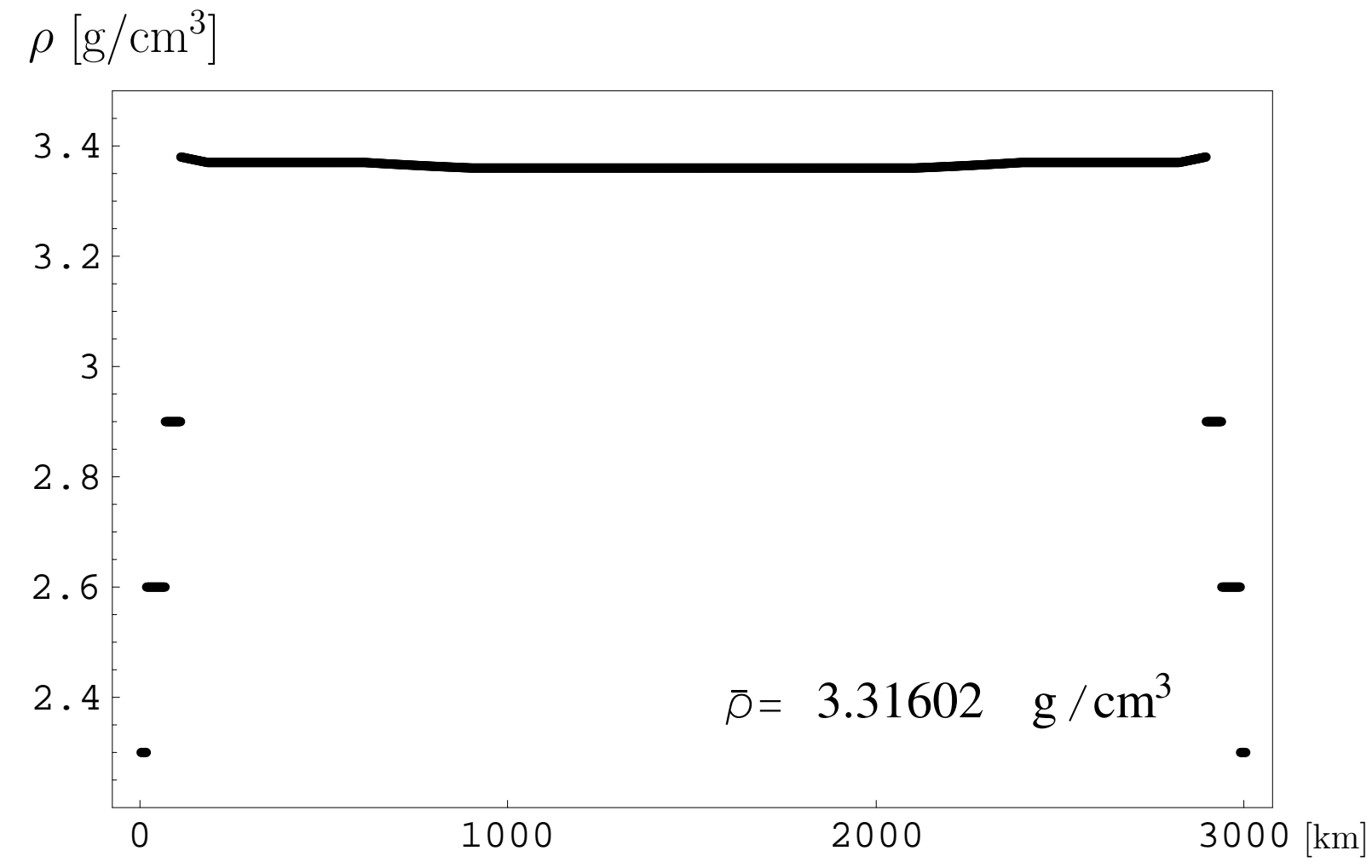

$\rho_{n}\left[\mathrm{~g} / \mathrm{cm}^{3}\right]$

(a) Matter profile

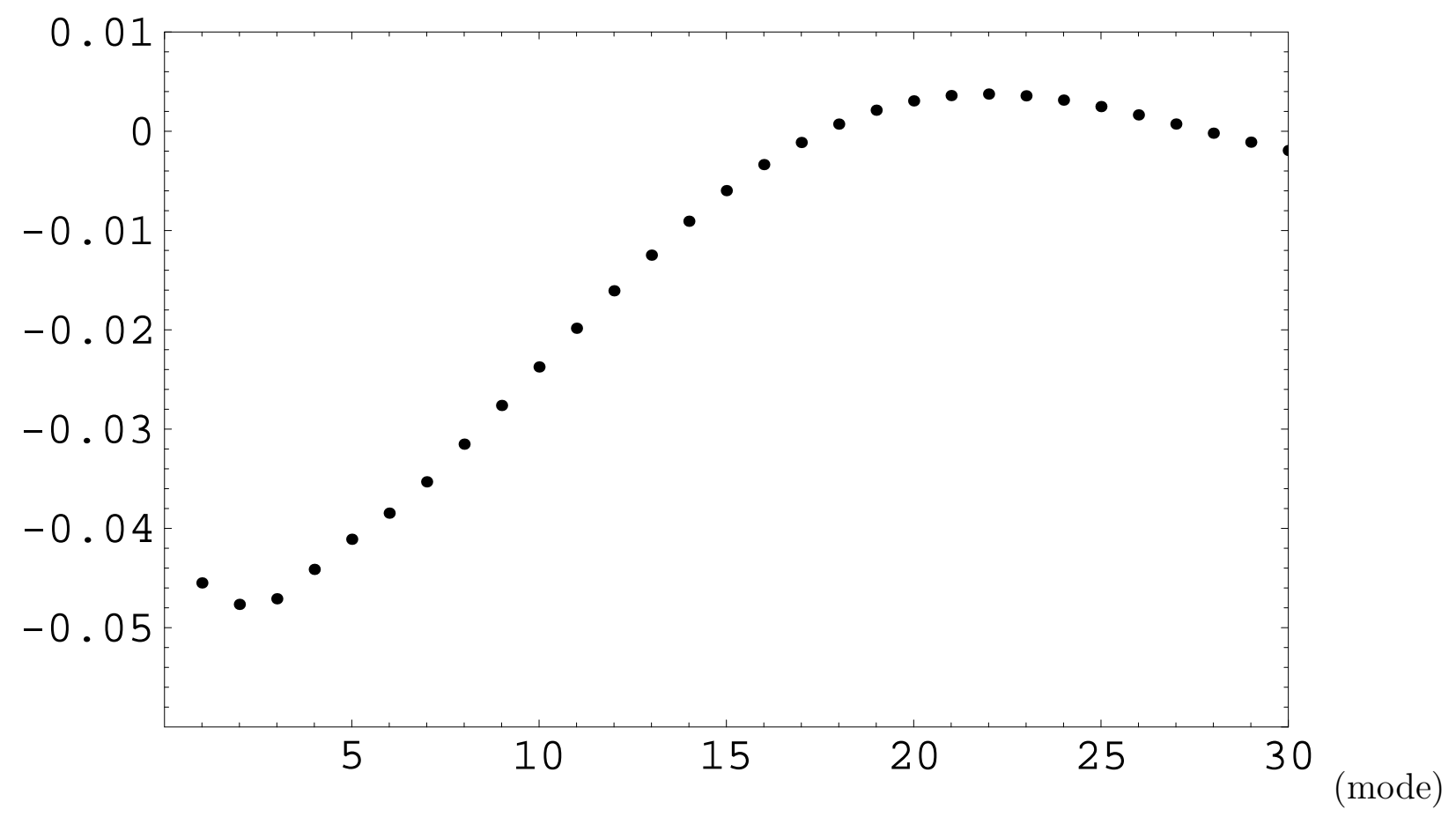

(b) Fourier Coeeficients

Figure 1: (a) Matter profile on the baseline and (b) its Fourier coefficients for $L=3000 \mathrm{~km}$. 


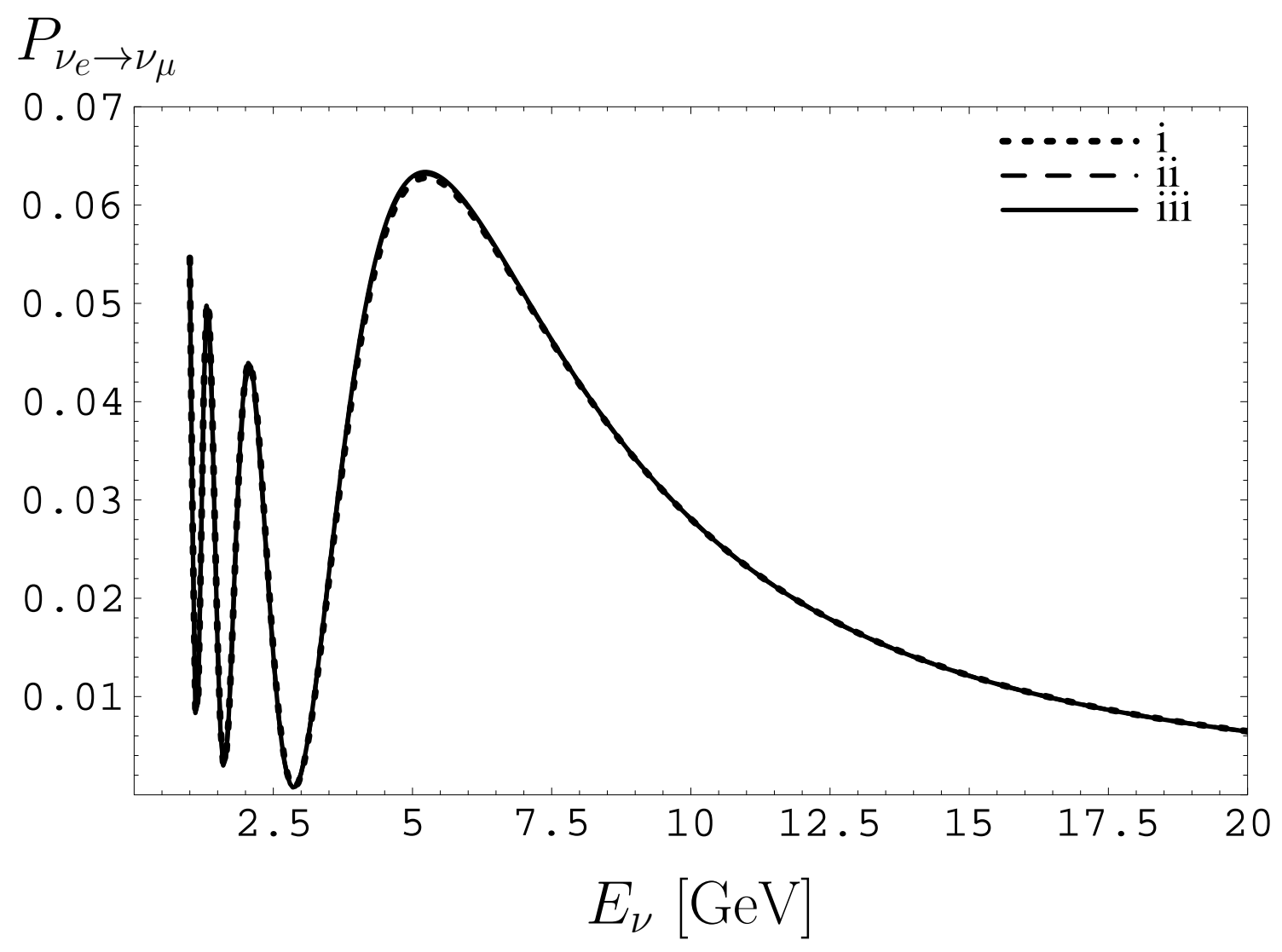

Figure 2: Transition probability $P_{\nu_{e} \rightarrow \nu_{\mu}}$ for $L=3000 \mathrm{~km}$. In this plot we set $\sin \omega=$ $1 / \sqrt{2}, \sin \psi=1 / \sqrt{2}, \sin \phi=0.1, \Delta m_{31}^{2}=3 \times 10^{-3} \mathrm{eV}^{2}, \Delta m_{21}^{2}=5 \times 10^{-5} \mathrm{eV}^{2}$ and $\delta=\pi / 2$. There are three lines corresponding to the matter profiles; (i) constant density (dotted line), (ii) eq.(17) with $\mathrm{N}=1$ (dashed line), and (iii) PREM matter profile in Fig.1 (solid line). These lines are quite similar to each other so that we cannot see those three lines in the graph. 


\section{Events per $\mathrm{GeV}$}
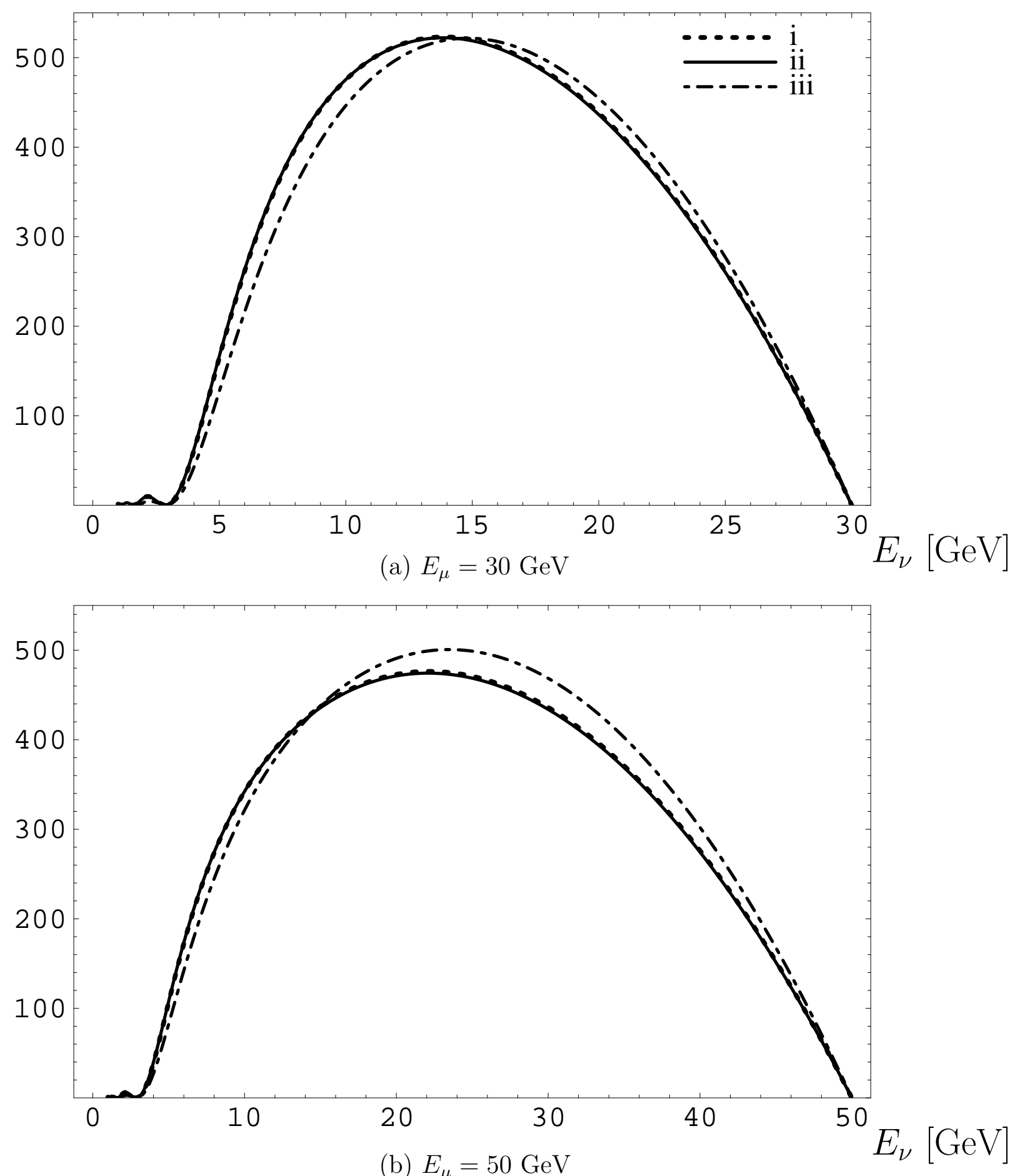

Figure 3: Differential event rate $N_{\nu_{e} \rightarrow \nu_{\mu}}$ for $L=3000 \mathrm{~km}$ with (a) $E_{\mu}=30 \mathrm{GeV}$ and (b) $E_{\mu}=50 \mathrm{GeV}$. The oscillation parameters are same as those in Fig.2 except $\delta$. There are three lines with respect to $\delta$ and the matter profiles;(i) $\delta=\pi / 2$ and constant density (dotted line), (ii) $\delta=\pi / 2$ and PREM matter profile (solid line), and (iii) $\delta=0$ and PREM matter profile (dash-dotted line). The line (iii) is different from the others. 
The matter profile based on the PREM and its Fourier coefficients are shown in Fig.4. We find that the first two coefficients are large. We argue, however, that the first Fourier coefficient among them contributes much more to the oscillation probability because the resonance condition (25) for $n= \pm 1$ can be satisfied in a neutrino factory.

In Fig.5, we compare the oscillation probabilities calculated with different matter profiles; (i) constant density (dotted line), (ii) eq.(7) with $N=1$ (dashed line), (iii) eq.(7) with $N=2$ (dash-dotted line), and (iv) the PREM matter profile in Fig. (⿴囗十 (solid line).

We see that the probability calculated using PREM (Fig.5 - (iv)) and another calculated using eq.(可) with $N=1$ (Fig.5 - (ii)) are very similar. However the probability calculated using the constant density matter profile (Fig. - - (i)) is quite different. Furthermore there is no significant difference between the probabilities calculated using eq.(7) with $N=1$ and $N=2$ (Fig. 5 - (ii) and (iii)). Thus we have to take into account a new parameter $a_{1}$ in the analysis of neutrino factories with the baseline $L=7332 \mathrm{~km}$. This new parameter, $a_{1}$, should be estimated by the experimental results.

To see the importance of $a_{1}$ more clearly, we show the event rate in Figs.6 using different $\delta$ and different matter profiles; (i) $\delta=\pi / 2$ and constant density approximation (dotted line), (ii) $\delta=\pi / 2$ and the matter profile (7) with $N=1$ (dashed line), (iii) $\delta=\pi / 2$ and the PREM matter profile (solid line), and (iv) $\delta=0$ and the PREM matter profile (dash-dotted line).

The distributions of the event rate in (Figs.6-(i)) and (Figs.6f(iii)) are quite different from each other. This means that the estimation of the event rate with constant density approximation doesn't work. In other words we cannot estimate the values of the parameters from the experimental data precisely without taking into account the contribution of $a_{1}$. On the other hand, the event rates in (ii) and (iii) are quite similar. If we can insist from Figs. 3 that we can observe CP violating effect, then we can insist from Figs.6 that we can observe the effect of $a_{1}$. Furthermore since the contribution of $a_{1}$ cannot be explained by other terms, we expect that we can estimate $a_{1}$ very well experimentally. We can study geophysics by neutrinos.

Consider the difference between Fig.6-(iii) and Fig.6-(iv). The effect of the CP violating phase on the event rate is so small that we cannot distinguish between the two theories that have different $\mathrm{CP}$ violating phase. Indeed the effect of $\delta$ is smaller than that of $a_{2}$ which is already beyond the experimental sensitivity.

\section{$3.3 \quad L=12000 \mathrm{~km}$}

In this case neutrinos penetrate the earth almost along its diameter. Neutrinos go through both the mantle and the core. Therefore the matter profile is very complicated. We expect that we can observe the higher modes in eq.(7).

The matter profile and the Fourier coefficients are given in Fig.7. From these figures we expect that not only $a_{1}$ but also the higher Fourier modes are relevant.

In Fig.8, we plot the transition probabilities corresponding to the matter profiles; (i) constant density (dotted line), (ii) eq.(7) with $\mathrm{N}=1$ (dashed line), (iii) eq.(7) with $\mathrm{N}=3$ (dash-dotted line), and (iv) PREM matter profile in Fig.7] (solid line). From Fig.8 we 

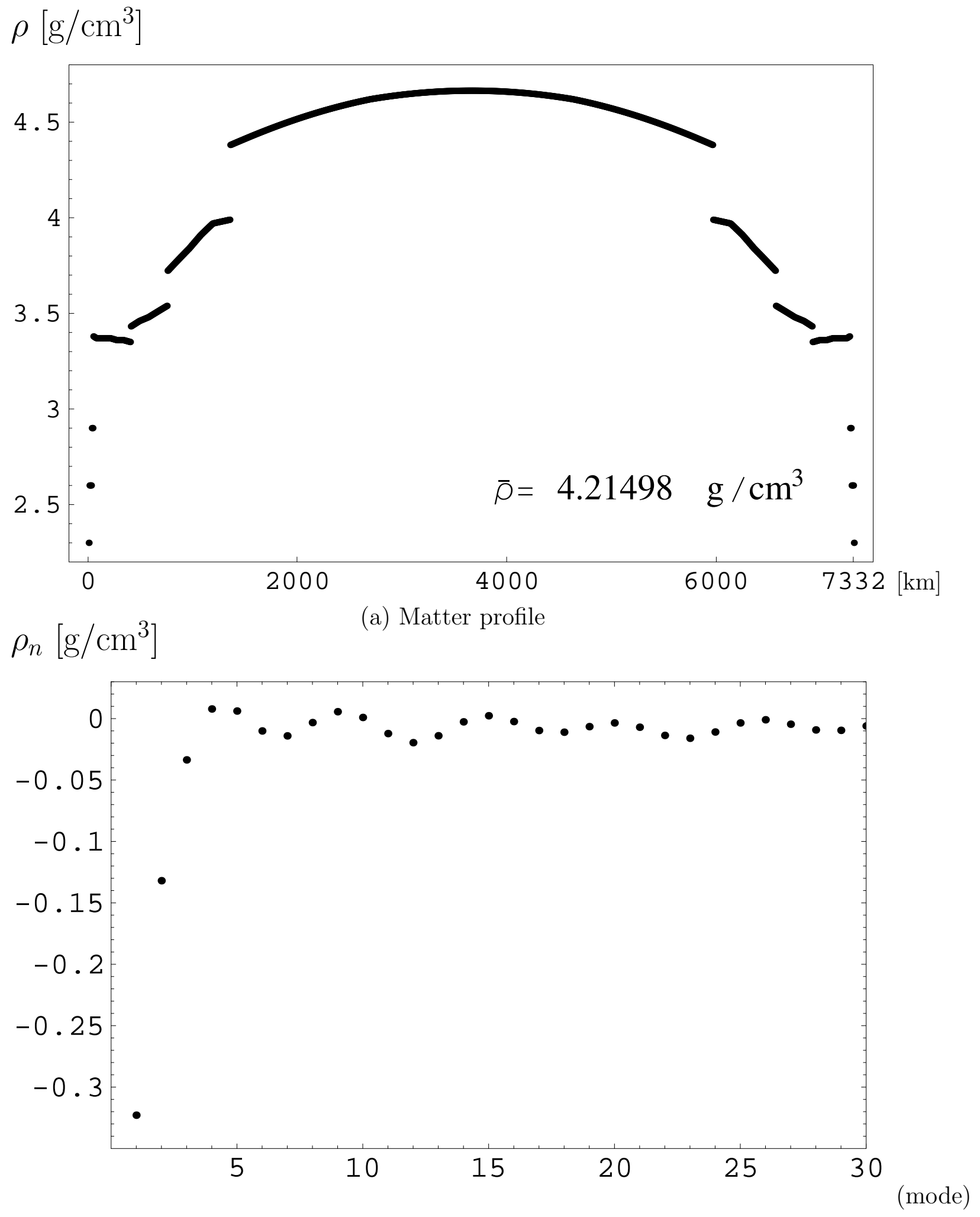

(b) Fourier Coeeficients

Figure 4: (a) Matter profile on the baseline and (b) its Fourier coefficients for $L=7332 \mathrm{~km}$. 


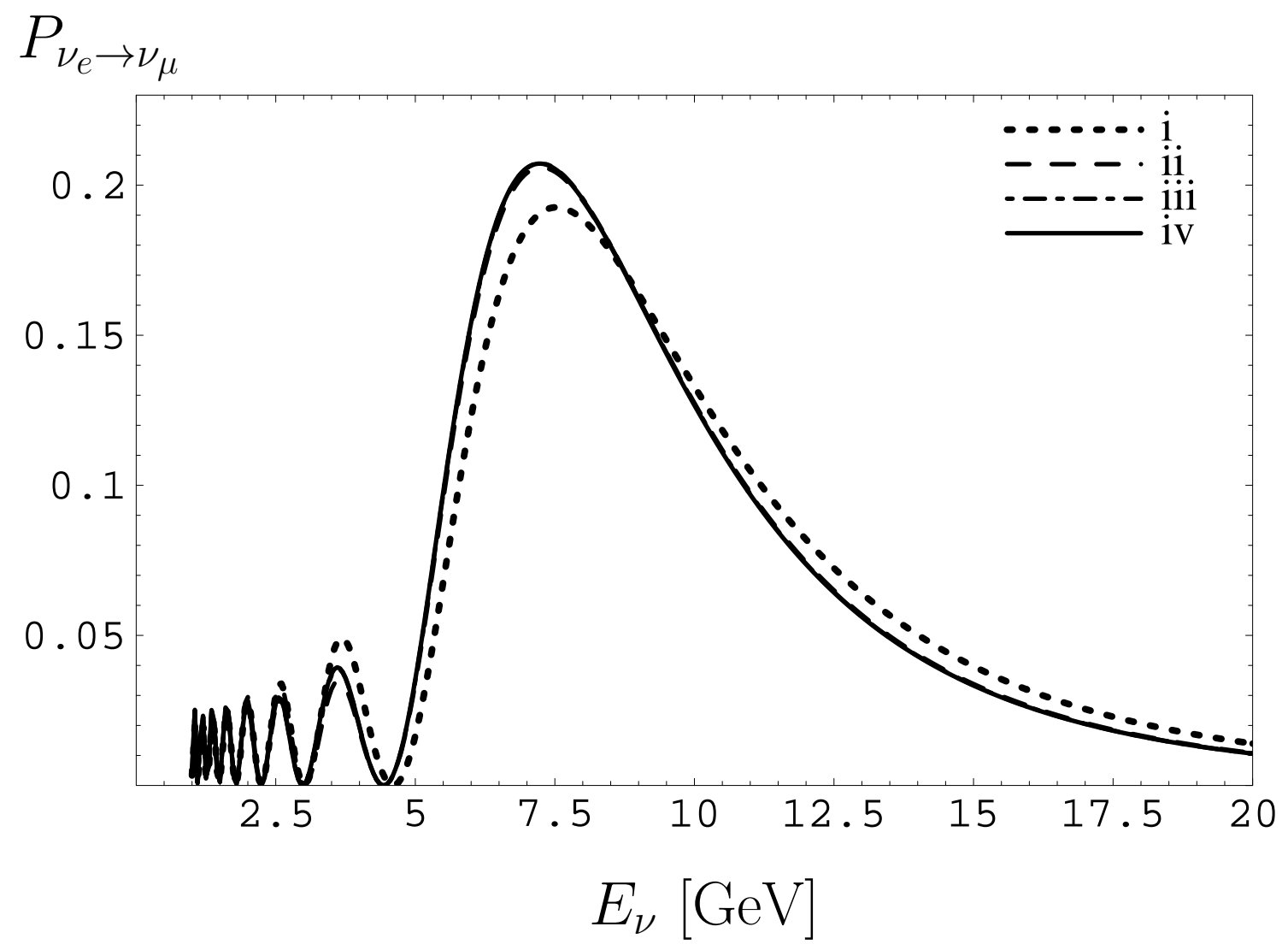

Figure 5: Transition probability $P_{\nu_{e} \rightarrow \nu_{\mu}}$ for $L=7332 \mathrm{~km}$. The oscillation parameters are the same as those in Fig.2. There are four lines corresponding to the matter profiles; (i) constant density (dotted line), (ii) eq.(17) with $\mathrm{N}=1$ (dashed line), (iii) eq.(7) with $\mathrm{N}=2$ (dash-dotted line), and (iv) PREM matter profile in Fig. (4 (solid line). The line (i) is quite different form the others while the other three lines show quite similar line shape. 
Events per GeV
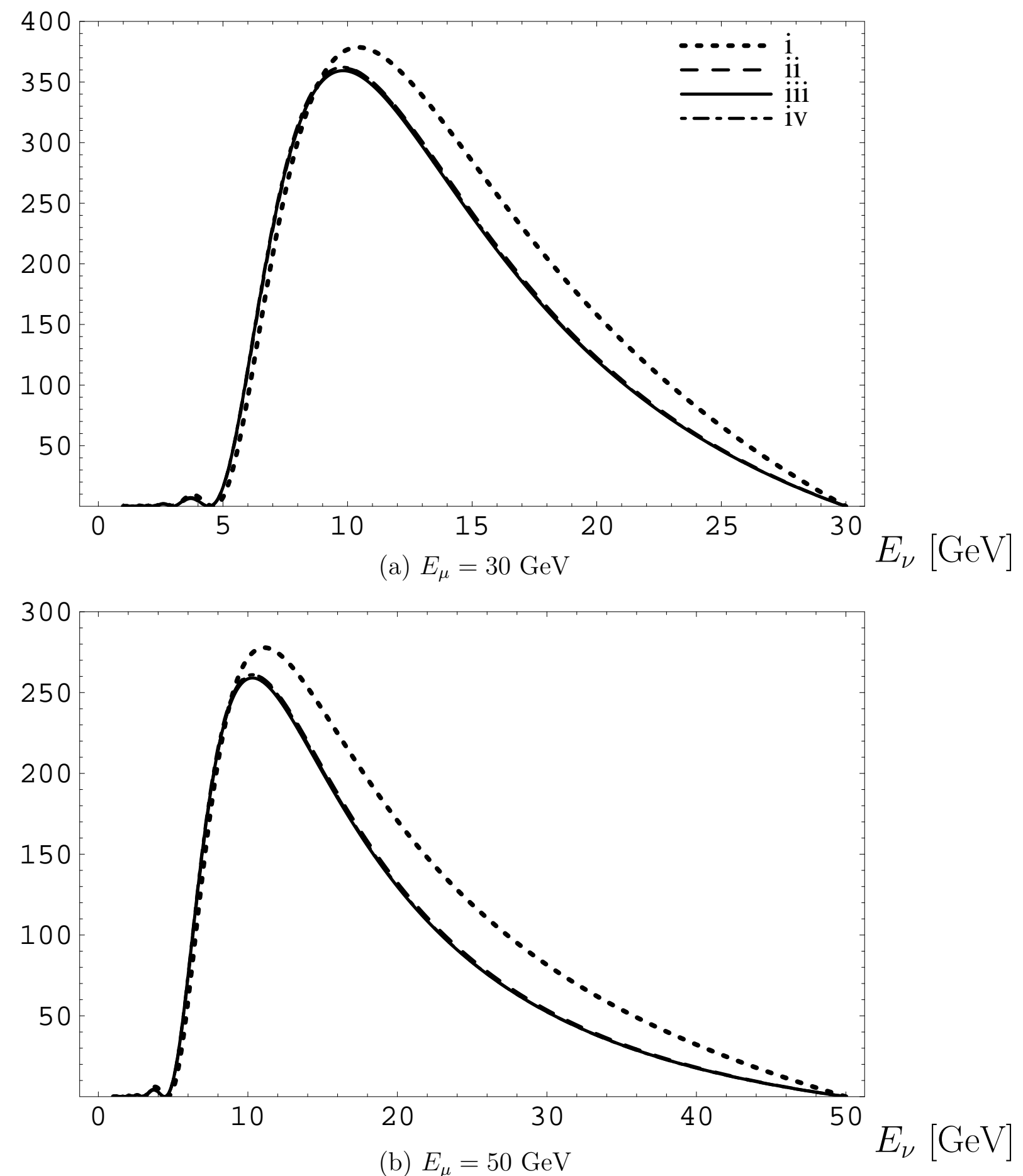

Figure 6: Differential event rate $N_{\nu_{e} \rightarrow \nu_{\mu}}$ for $L=7332 \mathrm{~km}$ with (a) $E_{\mu}=30 \mathrm{GeV}$ and (b) $E_{\mu}=50 \mathrm{GeV}$. The oscillation parameters are the same as those in Fig.2 except $\delta$. There are four lines with respect to $\delta$ and the matter profiles;(i) $\delta=\pi / 2$ and constant density ( dotted line), (ii) $\delta=\pi / 2$ and eq.(7) with $\mathrm{N}=1$ (dashed line), (iii) $\delta=\pi / 2$ and PREM matter profile (solid line), and (iv) $\delta=0$ and PREM matter profile (dash-dotted line). The line (i) is different from the others while the other three are quite similar. 


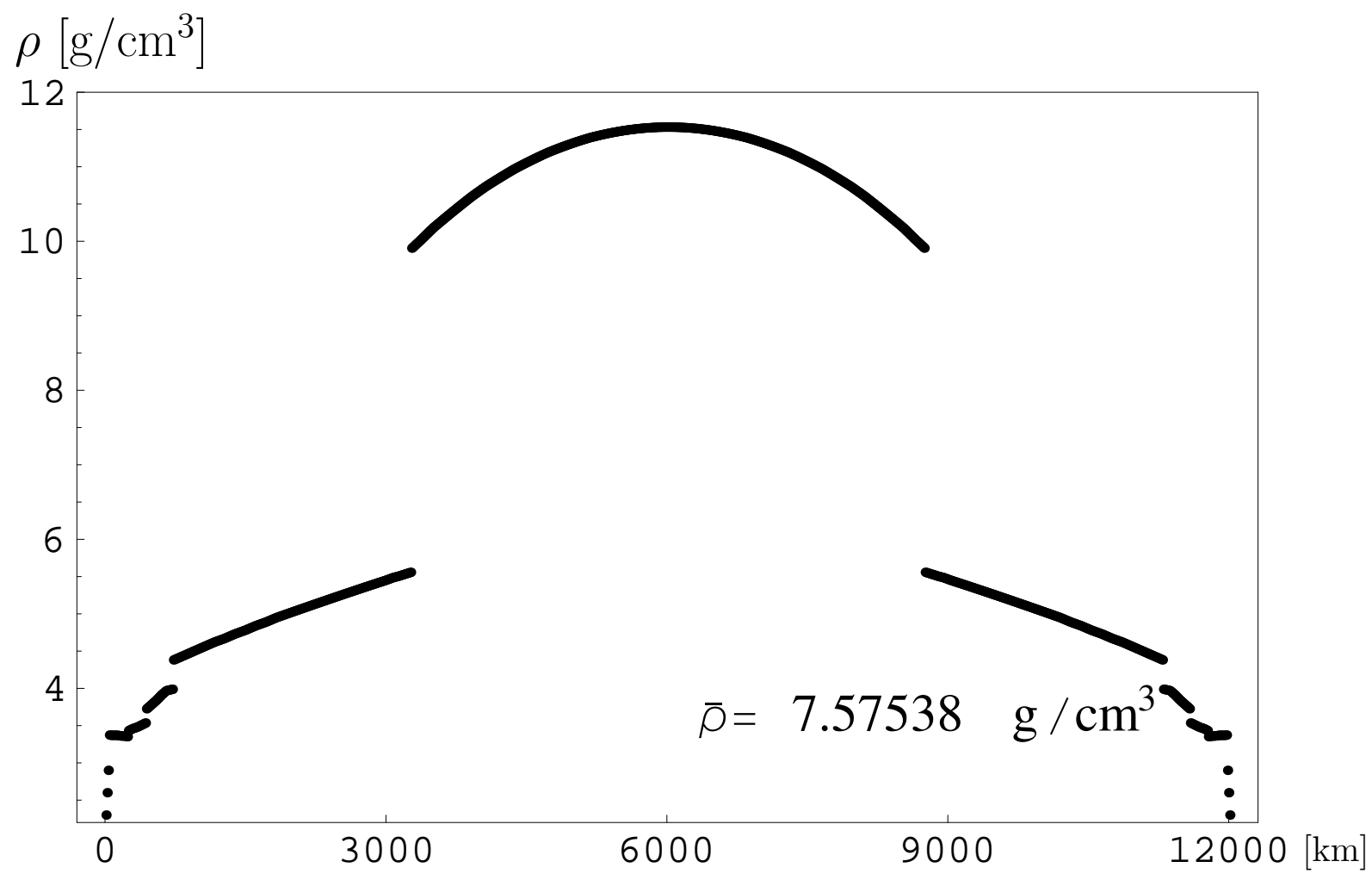

$\rho_{n}\left[\mathrm{~g} / \mathrm{cm}^{3}\right]$

(a) Matter profile

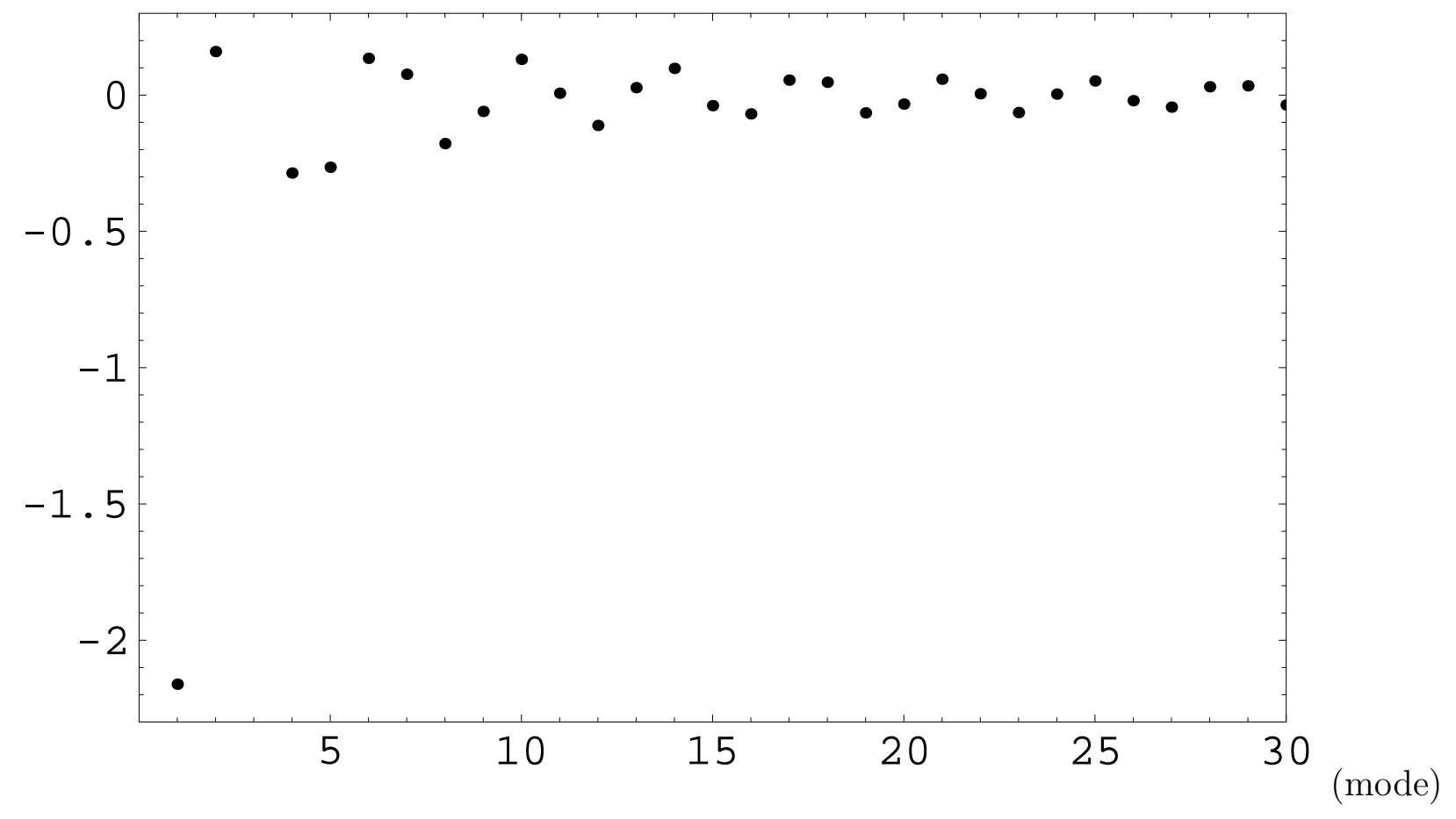

(b) Fourier Coeeficients

Figure 7: (a) Matter profile on the baseline and (b) its Fourier coefficients for $L=$ $12000 \mathrm{~km}$. 


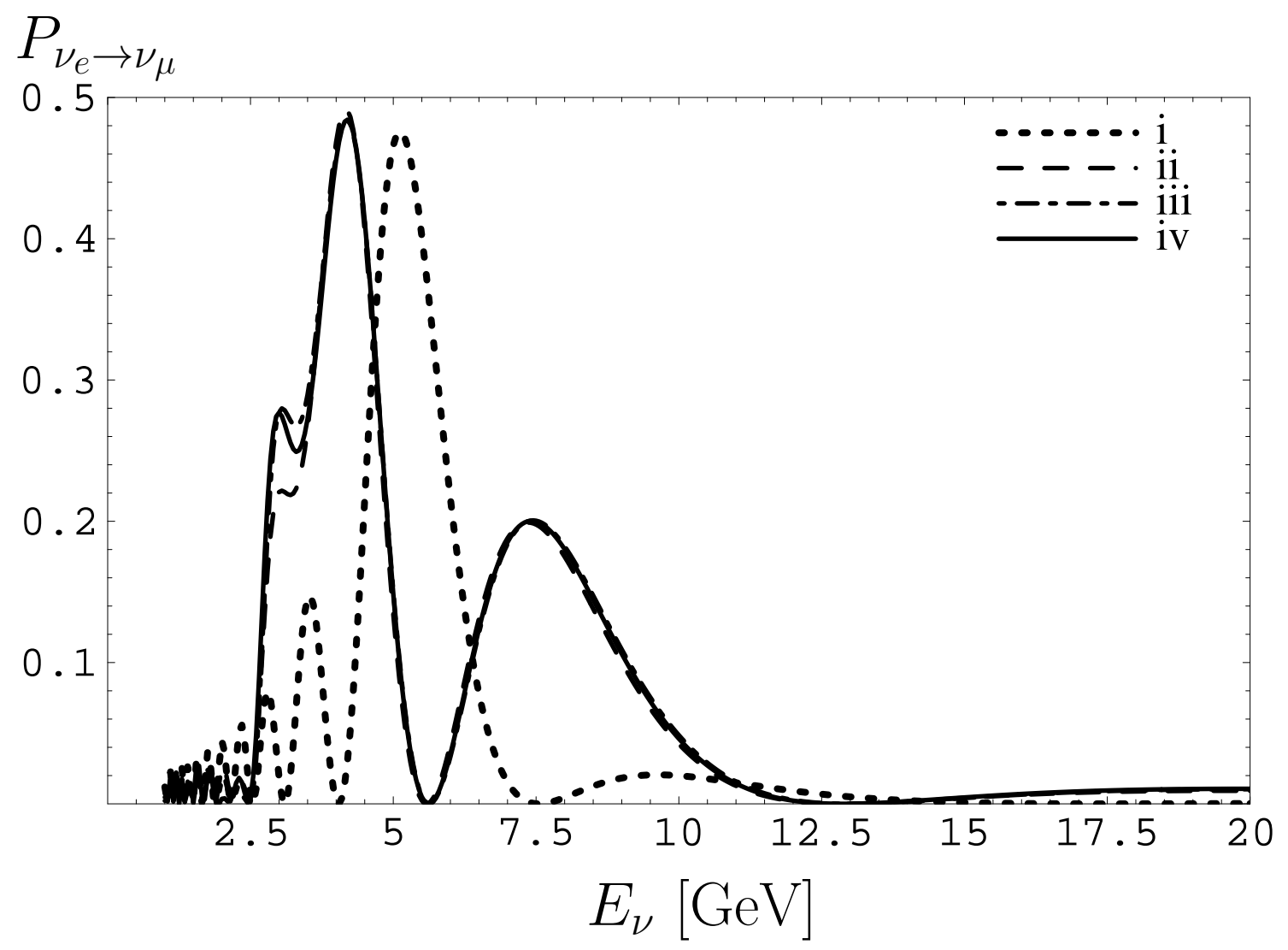

Figure 8: Transition probability $P_{\nu_{e} \rightarrow \nu_{\mu}}$ for $L=12000 \mathrm{~km}$. In this plot we use the same oscillation parameters as those in Fig.2. There are four lines corresponding to the matter profiles; (i) constant density (dotted line), (ii) eq.(17) with $\mathrm{N}=1$ (dashed line), (iii) eq.(7) with $\mathrm{N}=3$ (dash-dotted line), and (iv) PREM matter profile in Fig.7 (solid line). The line (i) is quite different from the others. The line (ii) is also different from the line (iV), while the line (iii) shows a quite similar shape with the line (iv). 


\section{Events per GeV}
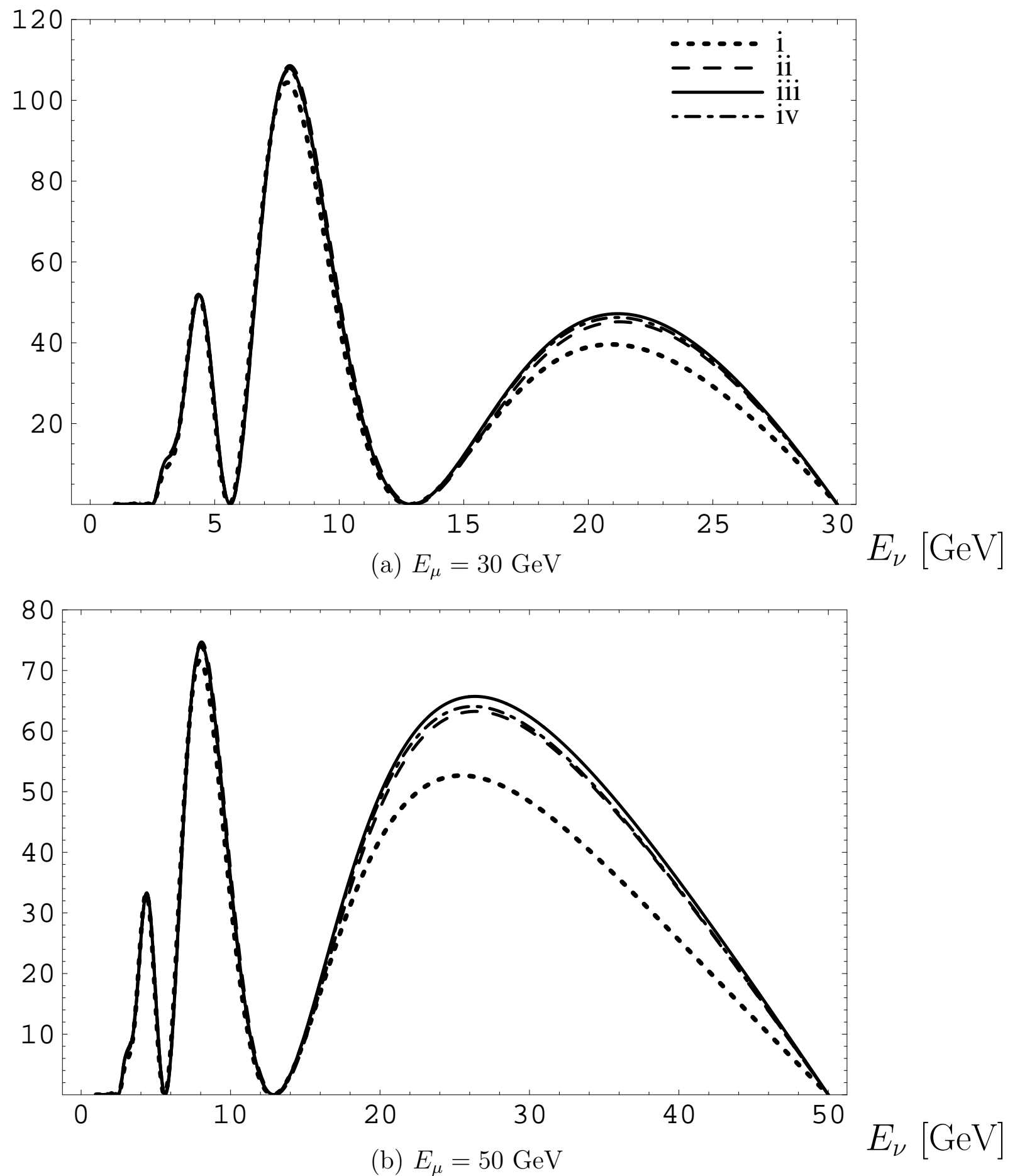

Figure 9: Differential event rate $N_{\nu_{e} \rightarrow \nu_{\mu}}$ for $L=12000 \mathrm{~km}$ with (a) $E_{\mu}=30 \mathrm{GeV}$ and (b) $E_{\mu}=50 \mathrm{GeV}$. The oscillation parameters are the same as those in Fig.2 except $\delta$. There are three lines corresponding to $\delta$ 's and the matter profiles;(i) $\delta=\pi / 2$ and eq.(7) with $N=1$ (dotted line), (ii) $\delta=\pi / 2$ and eq.(7) with $N=3$ (dashed line), (iii) $\delta=\pi / 2$ and PREM matter profile (solid line); (iv) $\delta=0$ and PREM matter profile (dash-dotted line). 
find that there are significant contributions to the oscillation probability from the higher modes. The probability calculated with the constant density matter profile (Fig.8-(i)) is quite different from that calculated with the PREM matter profile (Fig.8-(iv)). Even the probability calculated using the matter profile eq.(1) with $\mathrm{N}=1$ (Fig.8-(ii)) differs apparently from that with the PREM matter profile (Fig.8-(iv)). On the other hand we find that the matter profile eq.(7) with $\mathrm{N}=3$ mimic very well the PREM matter profile to calculate the oscillation probability.

We also plot the event rates in Figs.9. There the lines correspond to the following $\delta$ and matter profiles;(i) $\delta=\pi / 2$ eq.(7) with $N=1$ (dotted line), (ii) $\delta=\pi / 2$ and eq.(7) with $N=3$ (dashed line), (iii) $\delta=\pi / 2$ and PREM matter profile (solid line); (iv) $\delta=0$ and PREM matter profile (dash-dotted line). We see little discrepancy in Figs.9-(ii), (iii), and (iv), while there is a conceivable difference between Figs.95-(i) and the others. We find, therefore that the effect of $\delta$ is irrelevant with the oscillation physics while the first three modes of the Fourier coefficients have measurable contribution to it.

We examine more carefully whether the higher mode contribution is really measurable. The number of the necessary new parameters is quite dependent on the event rate of the experiment. We can find that there is conceivable difference between the lines of Figs.9-(i) and those of Figs.9-(ii)\& (iii). However this difference is not significant statistically in Fig.9 (a). As long as $N_{\mu} \times N_{\mathrm{kt}} \times \epsilon$ is less than $5 \times 10^{22}$, we need to introduce only $a_{1}$ as the theoretical parameter. On the other hand this discrepancy is very significant in Fig.9 (b). We can measure the contributions from the higher modes such as $a_{2}$ and $a_{3}$. We should introduce higher modes according to the expected event rate.

\section{Summary and discussion}

We considered how to deal with the matter profile effect. We proposed that the Fourier coefficients of the matter profile are used as the theoretical parameters. Using this method we can evaluate the size of the ambiguities in the estimate of the mixing parameters.

The perturbative solution for the evolution equation, eq.(24), implies that the higher Fourier modes are irrelevant. The introduction of the first few modes gives enough precision to the estimate of the event rate.

We saw the following three cases in detail numerically.

- $L=3000 \mathrm{~km}$. The matter profile effect itself is irrelevant. We can assume the matter density is constant.

- $L=7332 \mathrm{~km}$. We need to introduce $a_{1}$ as the theoretical parameter which should be measured experimentally. On the other hand the $\mathrm{CP}$ violating phase $\delta$ is irrelevant. We cannot determine the $\mathrm{CP}$ violating phase at this baseline.

- $L=12000 \mathrm{~km}$. We need to introduce higher modes as the theoretical parameters. However, since the event rate with this baseline length is significantly small, by using only $a_{1}$ we can estimate the theoretical parameters accurately within the precision 
of the experiment. Depending on the precision required, we must decide the number of coefficients to introduce.

In general the Fourier modes are relevant, when the baseline passes through the lower $\operatorname{mantle}(L \gtrsim 5000 \mathrm{~km})$. In this case we need to use the method developed in this paper to make an accurate analysis. In other words we can explore the interior of the earth in neutrino factories.

\section{Acknowledgments}

The authors are grateful to K. Harada and M. Koike for useful discussions. The work of J.S is supported in part by a Grant-in-Aid for Scientific Research of the Ministry of Education, Science and Culture, \#12047221, \#12740157.

\section{References}

[1] Super-Kamiokande Collaboration, Y.Fukuda et al., Phys. Rev. Lett. 81, 1562 (1998).

[2] for example, K. Nishikawa, INS-Rep-924 (1992).

S. Parke, Fermilab-Conf-93/056-T (1993), hep-ph/9304271.

see also http://cupp.oulu.fi/neutrino/.

[3] S. Geer, Phys. Rev. D57, 6989 (1998), erratum ibid. D59 (1999) 039903.

[4] V. Barger, S. Geer and K. Whisnant, Phys. Rev. D61 (2000) 053004.

[5] A. Cervera , A. Donini, M.B. Gavela, J.J. Gomez Cadenas, P. Hernandez, O. Mena and S. Rigolin, Nucl. Phys. B579 (2000) 17.

[6] V. Barger, S. Geer, R. Raja and K. Whisnant, Phys. Rev. D62 (2000) 013004; Phys .Rev. D62 (2000) 073002; Phys. Lett. B485 (2000) 379; hep-ph/0007181.

[7] A. De Rujula, M. B. Gavela and P. Hemandez, Nucl. Phys. B547, 21 (1999); A. Donini, M. B. Gavela, P. Hernandez and S. Rigolin, Nucl. Phys. B574 (2000) 23.

[8] K. Dick, M. Freund, M. Lindner, and A. Romanino, Nucl. Phys. B562 (1999) 29; A. Romanino, Nucl. Phys. B574 (2000) 675; M. Freund, M. Lindner, S. T. Petcov and A. Romanino, Nucl. Phys. B578 (2000) 27;

[9] M. Freund, P. Huber and M. Lindner, Nucl. Phys. B585 (2000) 105.

[10] Neutrino Factory and Muon Collider Collaboration (D. Ayres et al.), physics/9911009; C. Albright, et al. hep-ex/0008064.

[11] M. Campanelli, A. Bueno and A. Rubbia, hep-ph/9905240; A. Bueno, M. Campanelli and A. Rubbia, Nucl. Phys. B573 (2000) 27; Nucl. Phys. B589 (2000) 577. 
[12] M. Koike and J. Sato, Phys. Rev. D61 (2000) 073012.

[13] O. Yasuda, hep-ph/0005134.

[14] L. Wolfenstein, Phys. Rev. D17 (1978) 2369.

S. P. Mikheev and A. Yu. Smirnov, Sov. J. Nucl. Phys. 42 (1985) 913.

[15] A. M. Dziewonski and D. L. Anderson, Phys. Earth Planet. Inter. 25 (1981) 297.

[16] M. Koike and J. Sato, Mod. Phys. Lett. A14 (1999) 1297.

[17] P. I. Krastev and A. Yu. Smirnov, Phys. Lett. B226 (1989) 341.

[18] M. Koike, T. Ota and J.Sato, in preparation. 